CASE REPORT (20 Jan. 2016)

\title{
Post-traumatic Digital Gangrene Associated with Epinephrine Use in Primary Raynaud's Phenomenon: Lesson for the Future
}

\author{
Carlson-Babila Sama ${ }^{1}$
}

\begin{abstract}
BACKGROUND: Following digital surgical procedures, the ensuing post-operative course may be complicated by the presence of underlying ischaemic or vasospastic process. In the presence of such conditions, post-operative ischaemic changes may be further exacerbated with the use of local anaesthetics in combination with epinephrine.

CASE DETAILS: We report a 21 year-old female who presented with an amputated fifth digit due to a rapidly spreading gangrene which started immediately after the surgical repair of a traumatic laceration which was infiltrated with a pre-mixed solution of lignocaine and epinephrine 3 hours earlier. The patient's final diagnosis was epinephrine-associated digital gangrene in the background of primary Raynaud's Phenomenon (RP).

CONCLUSION: The author reports this case in order to reiterate the importance of thorough clinical evaluation prior to the use of epinephrine in digital anaesthesia as well as to increase awareness on how primary RP can be complicated by gangrene.
\end{abstract}

KEYWORDS: Digital gangrene, trauma, epinephrine, Raynaud's phenomenon

DOI: http://dx.doi.org/10.4314/ejhs.v26i4.13

\section{INTRODUCTION}

Raynaud's phenomenon (RP) represents a challenging disease for physicians due to the variability in its presentation, wide range of medical therapies and significant risk of morbidity(1). It refers to reversible spasm of peripheral arterioles in response to cold temperatures or emotional stress often seen in distal digits but may also involve other sites such as the ear, the nose and the tongue (2). $\mathrm{RP}$ is characterized by a triphasic response involving pallor due to vasoconstriction of precapillary muscular arterioles, cyanosis due to venous pooling and deoxygenation of venous blood and erythema due to reactive hyperemia associated with throbbing pain $(1,2)$.

\section{CASE REPORT}

A 26 year-old female student presented to our emergency room (ER) with an amputated left fifth digit and an ongoing ischaemic process at the base of her left fourth digit (Figure 1). Three hours earlier, she had sustained a traumatic laceration extending from the palmar surface of the fifth digit to the base of the fourth digit. It was sutured under local anaesthesia by infiltration of a pre-mixed solution of $1 \%$ lignocaine with epinephrine at a local health centre. She reported that almost immediately after the procedure, she experienced intense pain, and the area started to turn black. The gangrene spread rapidly over a 2-hour period to involve the entire fifth digit which was

${ }^{1}$ Galactic Corps Research Group (GCRG) and Department of Clinical Sciences, Faculty of Health Sciences, University of Buea, Buea, South-West Region, Cameroon

Corresponding Author: Carlson-Babila Sama, Email: sama.carlson@ yahoo.com 
subsequently amputated. She then presented to our ER 30 minutes after amputation for higher level of care due to concerns that she might lose the fourth digit too as its base had just started to turn black.

She was not on routine medication and had no known chronic illness but described a classical intense spasmic digital pain associated with change in colour when fingers were exposed to cold which started 9 months earlier.

On examination, she was anxious but vital signs were stable. Her left hand showed the base of a poorly amputated fifth digit and a dark-blue discoloration at the base of the fourth digit with decreased perception to light touch and delayed capillary refill time at its tip. All peripheral pulses were equal and normal. Initial workup including full blood count, urinalysis, erythrocyte sedimentation rate, C-reactive protein, serum complements ( $\mathrm{C} 3$ and $\mathrm{C} 4)$, serum electrophoresis, thyroid, kidney and liver function tests were all within normal levels. Screening for hepatitis B and $\mathrm{C}$ viruses were negative. X-ray of the hands ruled out subcutaneous calcification. Normal results were obtained on nail fold capillaroscopy and Doppler arterial ultrasonography. Immunological tests including extractable nuclear antigen, antinuclear antibody, anti-neutrophil cytoplasmic antibodies, rheumatoid factor, anti-cardiolipin antibodies and lupus anticoagulant were negative. Based on the above findings, we reached a diagnosis of primary RP, and she was treated with vasodilatory therapy and antibiotics. The ischaemic process halted immediately and healing with minimal scarring at the site was achieved within 2 weeks. Routine weekly follow-up over a 7 weeks period found her to be in good health.

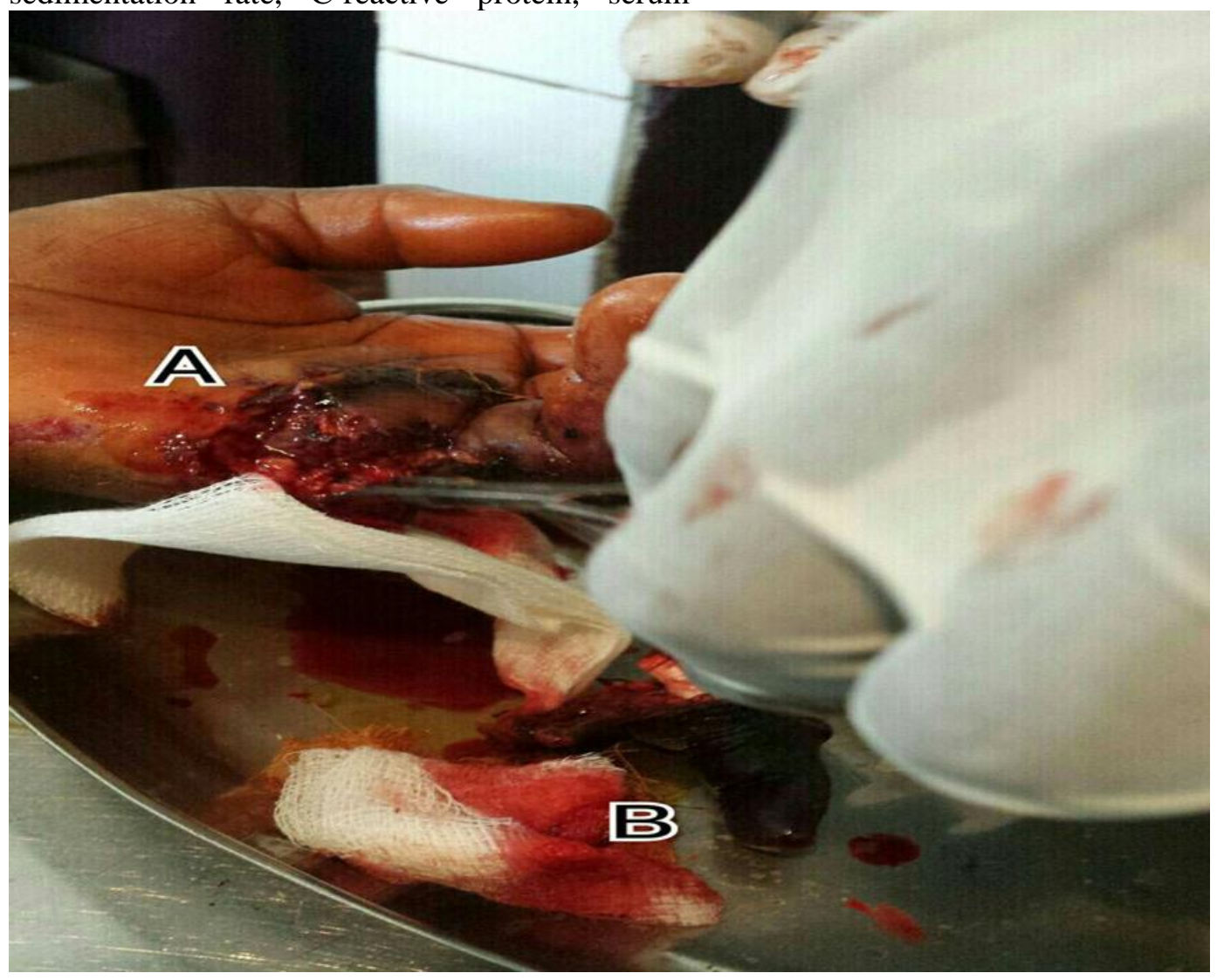

Figure 1: A shows the base of the poorly amputated left fifth digit and the ongoing is chaemic process at the base of the fourth digit; $\boldsymbol{B}$ illustrates gangrenous amputated fifth digit 


\section{DISCUSSION}

Rapidly spreading gangrene following a digital surgical procedure as observed in this case is a rare scenario. Thus, it is imperative that clinicians seek for an alternative explanation when such events occur in order to prevent future significant morbidity.

Raynaud's phenomenon was first recognized in 1862 by Maurice Raynaud. It refers to a reversible, episodic vasospasm of digital arteries, pre-capillary arterioles and cutaneous arteriovenous shunts in response to cold or stress resulting in pallor, cyanosis and occasionally subsequent ischaemia $(1,3)$. Various vascular and neural factors are thought to play a role in its occurrence by favouring vasoconstriction and somewhat impairing vasodilatory mechanisms in a complex interplay (2-4). Primary RP is also referred to as Raynaud's disease is idiopathic, while secondary RP occurs in association with an underlying disease, usually a connective tissue disease, neurovascular, haematological, or druginduced disorders (1). Spasmic episodes are a rare complication, often mild, if it develops in primary RP. In secondary RP, episodes are usually severe and may lead to digital ulcers, tissue necrosis and gangrene $(1,4)$.

Epinephrine (adrenaline) is a strong alphaand beta-receptor agonist and plays a central role in the short-term stress reaction. It results in the activation of alpha-receptors in digital arteries leading to a vasoconstrictory response. Digital arteries are terminal or end arterioles, and this vasoconstriction can potentially lead to ischemia and consequent gangrene (5). The use of epinephrine in digital anaesthesia is still clouded in controversy due to this inherent risk of causing vascular insufficiency and digital ischaemia. However, several studies report the use of a commercial lidocaine-epinephrine mixture under such circumstances with no adverse outcomes (6$9)$. In a recent study, Junior et al concluded that the use of a mixture of lidocaine with epinephrine for digital nerve block in hand surgery, at a concentration of $1: 100,000$ or less, is a safe technique to achieve local anaesthesia, without resultant complications relating to gangrene or systemic absorption (6). In our patient, the possible mechanism of the ensuing gangrene appears to be an augmentation of the pre-existing

enhanced adrenergic vasoconstrictive response in RP due to local infiltration rather than a digital nerve block of the epinephrine mixed with lignocaine prior to the surgical repair of the laceration. More so, the concentration of epinephrine in the mixture was unknown. Thus, it is possible that exponentially larger doses of epinephrine were injected.

It is hoped that this case will increase awareness amongst clinicians on the importance of carrying out a thorough history and clinical evaluation to identify pre-existing underlying vasospastic disorders in all patients prior to the use of epinephrine in digital surgical procedures. In the absence of such disorders, digital nerve block with known low dose concentrations of epinephrine in local anaesthesia is preferable over local infiltrations. Due to the risk of severe vasoconstriction, the use of epinephrine for such procedures should be avoided in patients with a known vascular compromize such as RP. It is paramount that physicians remain alert during such procedures so as to quickly recognize and treat potential postoperative gangrene. Since timely intervention would have probably salvaged the amputated digit.

\section{ACKNOWLEDGEMENTS}

Sincere thanks goes to the staff of the Douala General Hospital, Douala, Cameroon, especially to the departments of Rheumatology and Orthopaedic Surgery for their enormous assistance in the work-up of this patient.

\section{REFERENCES}

1. Villanueva $H$, Tijunelis $M$, Shapiro $S$, Henderson S. Loss of Digits after Trauma in a Patient with Systemic Lupus Erythematosus. WestJEM. 2008;9:43-5.

2. Saigal R, Kansal A, Mittal M, Singh Y, Ram H. Raynaud's Phenomenon. JAPI. 2010;58:309-13.

3. Wigley F. Raynaud's Phenomenon. $N$ Engl $J$ Med. 2002;347(13):1001-8.

4. Bakst R, Merola J, Franks A. Raynaud's phenomenon: pathogenesis and management. J Am Acad Dermatol. 2008;59:633-53. 
5. Krunic A, Wang L, Soltani K, Weitzul S, Taylor R. "Digital anesthesia with epinephrine: an old myth revisited"Journal of the American Academy of Dermatology. 2004;51(5):755-9.

6. Junior R, Costa J, Carmo J. Use of adrenalin with lidocaine in hand surgery. Rev Bras Orthop. 2014;49(5):452-60.

7. Chowdhry S, Seidenstricker L, Cooney D. Do not use epinephrine in digital blocks: myth or truth? Part II. A retrospective review of 1,111 cases. Plast Reconstr Surg. 2010;126:2031-4.

8. Denkler K. comprehensive review of epinephrine in the finger: to do or not to do. Plast Reconstr Surg. 2001;108:111-24.

9. Waterbrook A, Germann C, Southall J. Is epinephrine harmful when used with anesthetics for digital nerve blocks? Ann Emerg Med. 2007;50(4):472-5. 